\title{
Study on the Potential Toxicity of a Thymoquinone-Rich Fraction Nanoemulsion in Sprague Dawley Rats
}

\section{Zaki Tubesha ${ }^{1,2}$, Mustapha Umar Imam ${ }^{1}$, Rozi Mahmud ${ }^{3}$ and Maznah Ismail ${ }^{1,2, *}$}

1 Laboratory of Molecular Biomedicine, Institute of Bioscience, Universiti Putra Malaysia, 43400 Serdang, Selangor Darul Ehsan, Malaysia

2 Department of Nutrition and Dietetics, Faculty of Medicine and Health Sciences, Universiti Putra Malaysia, 43400 UPM, Serdang, Selangor, Malaysia

3 Department of Imaging, Faculty of Medicine and Health Sciences, Universiti Putra Malaysia, 43400 UPM, Serdang, Selangor, Malaysia

* Author to whom correspondence should be addressed; E-Mail: maznah@medic.upm.edu.my; Tel.: +603-8947-2115; Fax: +603-8947-2116.

Received: 27 May 2013; in revised form: 18 June 2013 / Accepted: 19 June 2013 /

Published: 26 June 2013

\begin{abstract}
Toxicological studies constitute an essential part of the effort in developing an herbal medicine into a drug product. A newly developed thymoquinone-rich fraction nanoemulsion (TQRFNE) has been prepared using a high pressure homogenizer. The purpose of this study was to investigate the potential acute toxicity of this nanoemulsion in Sprague Dawley rats. The acute toxicity studies were conducted as per the OECD guidelines 425, allowing for the use of test dose limit of $20 \mathrm{~mL}$ TQRFNE (containing $44.5 \mathrm{mg}$ TQ) $/ \mathrm{kg}$. TQRFNE and distilled water (DW) as a control were administered orally to both sexes of rats on Day 0 and observed for 14 days. All the animals appeared normal, and healthy throughout the study. There was no observed mortality or any signs of toxicity during the experimental period. The effects of the TQRFNE and DW groups on general behavior, body weight, food and water consumption, relative organ weight, hematology, histopathology, and clinical biochemistry were measured. All the parameters measured were unaffected as compared to the control (DW) group. The administration of $20 \mathrm{~mL}$ TQRFNE / kg was not toxic after an acute exposure.
\end{abstract}

Keywords: Sprague-Dawley rats; nanoemulsion; thymoquinone rich fraction; acute oral toxicity; histopathology; hematology 


\section{Introduction}

Drug efficacy and bioavailability can be severely limited by poor aqueous solubility and some drugs also show side effects due to their poor solubility [1]. Moreover, it is widely recognized that up to $60 \%$ of new drugs in the developmental stage are water insoluble [2]. Therefore, the improvement of drug solubility and thereby its bioavailability remains one of most challenging aspects of drug development process [1]. In the past few decades, considerable attention has been focused on the development of novel drug delivery systems for herbal drugs [2]. However some limitations during consumption of herbal extracts or plant bioactives like instability in highly acidic $\mathrm{pH}$ and liver metabolism led to drug levels below therapeutic concentration in the blood resulting in less or no therapeutic effect [3]. Hence, encapsulation of plant extracts or its bioactives minimizes their degradation or presystemic metabolism, and serious side effects by accumulation of drugs to the non-targeted areas and improves the ease of administration in the pediatric and geriatric patients [4].

A wide variety of drug carriers including nanoemulsions, microemulsions, liposomes, solid lipid nanoparticles, microspheres, and self-microemulsifying drug-delivery systems have been studied to improve the therapeutic efficacy of hydrophobic drugs/nutraceuticals by enhancing the bioavailability and tissue-targeting ability [5]. Lipid nanoemulsions containing oil from medicinal plants or hydrophobic drugs have been shown to improve drug solubility, reduce side effects of various potent drugs, increase the bioavailability of drugs, and to prolong the pharmacological effects in comparison to conventional formulations such as conventional emulsions [6]. Hence, nanoemulsions can be used as novel formulations in many areas including pharmaceutics, cosmetics, food technology, and other sectors [7]. Despite the beneficial effects of the nanocarriers on living cells, undesirable effects may occur. Hence, before marketing a new nanocarrier, extensive toxicity tests are required before it is deemed "safe" for marketing [8]. When sufficient toxicological data from in vitro and in vivo experiments are available, the pharmaceutical then may be applied to a human population. Therefore, data obtained from such tests are then used to extrapolate the doses and effects on humans [9]. Determination of acute oral toxicity is usually an initial screening step in the assessment and evaluation of the toxic characteristics of newly developed compounds [10].

Nigella sativa oil and its main active constituent, thymoquinone (TQ), are extensively reported to exhibit protective effects against many diseases largely attributable to its high antioxidant activity $[11,12]$. However, thymoquinone-rich fraction (TQRF) and TQ are lipophilic substances with limited absorbance in living system. Hence, nanoemulsions from TQRF and TQ were developed in attempt to increase their absorbance and bioavailability. Regarding the safety of N. sativa; its seed powder did not produce any toxic effects at very high doses ( $28 \mathrm{gm} / \mathrm{kg}$ orally) in rabbits; its oil was also safe when given orally to rats $\left(\mathrm{LD}_{50}\right.$ of $\left.28.8 \mathrm{~mL} / \mathrm{kg}\right)$; and oral TQ was also found to be safe ( $\mathrm{LD}_{50}$ of $\left.2.4 \mathrm{~g} / \mathrm{kg}\right)$ [13]. However, there are toxicological concerns and ethical issues that come with nanomedicine and they have to be addressed alongside the benefits. Moreover, nanotoxicology is now becoming a very important field in view of the unique physicochemical properties of nanomaterials that may lead to adverse biological effects on occupational, environmental and consumer matters [14]. Hence, despite the wide margin of safety of $N$. sativa oil, the toxicity study of newly developed TQRF nanoemulsion (TQRFNE) is very important. 


\section{Results and Discussion}

\subsection{Clinical Signs and Necropsy Findings}

This study was designed to assess the acute oral toxicity of TQRFNE which was administered by oral gavage to male and female rats. No deaths or obvious clinical signs were found in any groups throughout the experimental period. Physical observation of the treated rats throughout the study indicated that none of them showed signs of toxicity such as skin, fur, eyes, mucus membrane and behavioral changes, tremors, salivation, diarrhea, sleep and coma. Normal body weight gains were observed during the study period compared to the control group. No abnormal gross findings were observed in the necropsies of any of the animals.

\subsection{Body Weight, Food and Water Consumption during the 14 Days}

The body weights of the TQRFNE and control rats are shown in Figure 1. There were gradual increases in body weight of TQRFNE and control rats. After the first week of the study, the body weight increases of all groups were not significant for both sexes compared to day 0; similar results were also shown for female rats after 2 weeks (day of sacrifice). On the other hand, both male groups (TQRFNE and control) exhibited a significant body weight increase after 2 weeks. At the end of the study, the percentages increase in body weight for male of treatment rats were found to be 9.83 and $10.37 \%$, and for female rats $7.89 \%$ and $8.87 \%$ of distilled water (DW) and TQRFNE, respectively. The food and water consumption of the treated rats were also not significantly different compared to the control rats measured throughout the study (Figure 2). The progressive increase in body weights of rats during the study period may indicate the improvement in the nutritional state of the animals. The growth response effect could be as a result of increased food and water intake.

Figure 1. Effects of oral administration of thymoquinone-rich fraction nanoemulsion (TQRFNE) and distilled water (DW) on body weights of male and female rats $(n=5)$. For each gender $\mathrm{P}$ values $<0.05$ were considered as significant using one way ANOVA followed by Least Significant Difference (LSD). Asterisks on bars of $7^{\text {th }}$ and $14^{\text {th }}$ days denote significant difference compared to day 0 in each gender.

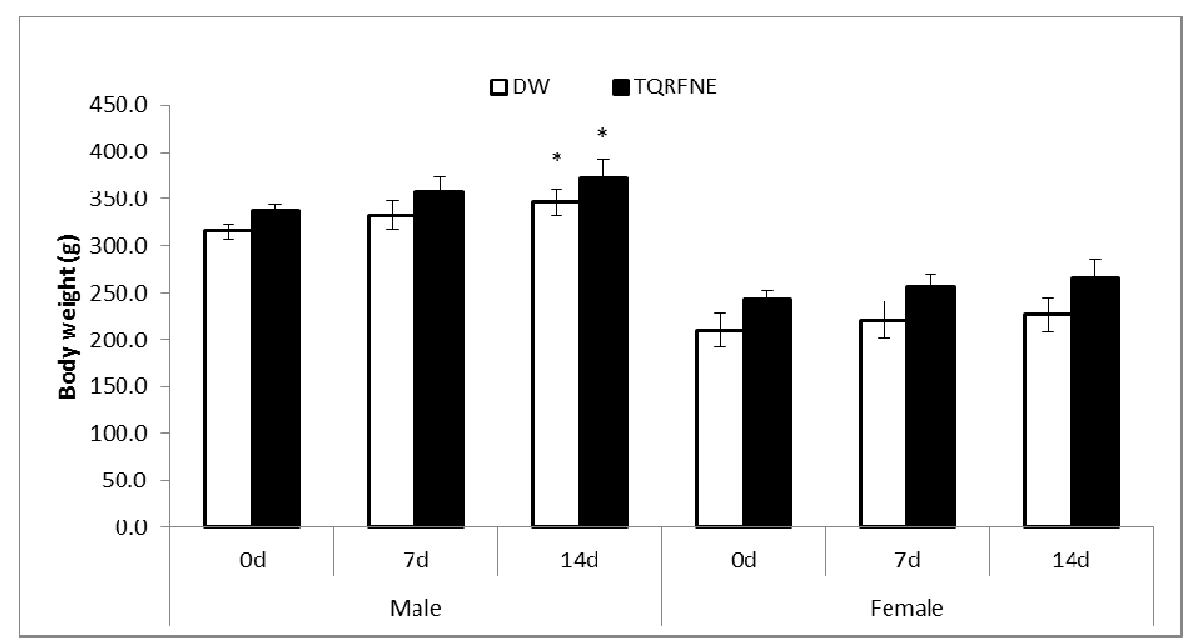


Figure 2. Effects of oral administration of thymoquinone-rich fraction nanoemulsion (TQRFNE) and distilled water $(\mathrm{DW})$ on daily food $(\mathrm{g} / \mathrm{rat} / \mathrm{d})$ and water $(\mathrm{ml} / \mathrm{rat} / \mathrm{d})$ consumption of male and female rats $(n=5)$. Values are expressed as mean \pm SD. P values $<0.05$ were considered as significant using one way ANOVA followed by Least Significant Difference (LSD). For each gender, there was no significant difference $(p \geq 0.05)$ compared to the control group (DW). FC: Food consumption, WC: Water consumption.

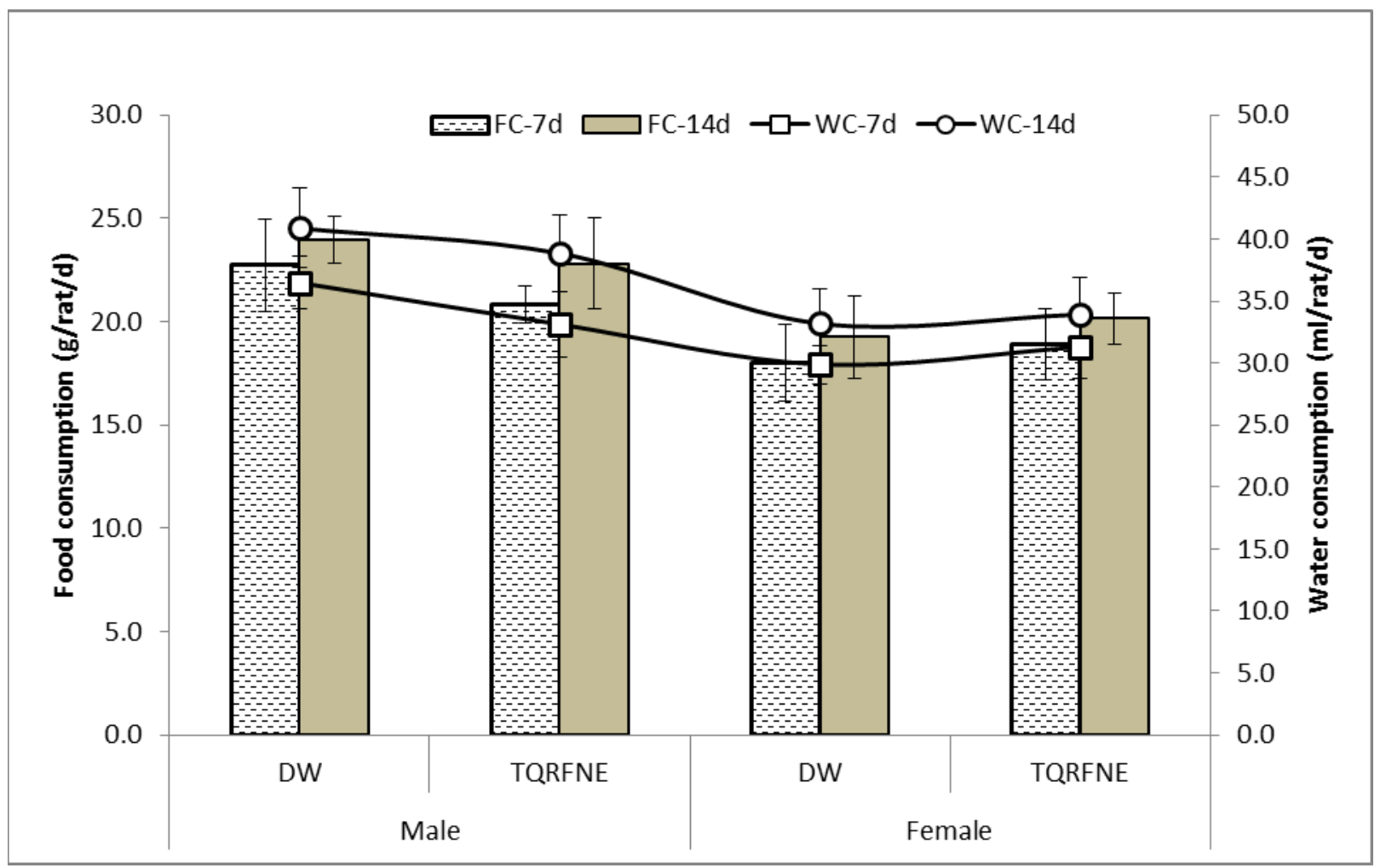

\subsection{Relative Organ Weights (ROW) of Male and Female Rats}

For prediction of basal metabolism, use of organ weights and ROW seem to be promising in safety and toxicity assessment [15]. There were no significant $(p>0.05)$ differences between ROW of treated and control rats (Table 1). The progressive increase in body weights and organ weights of rats during the study period may indicate the improvement in the nutritional state of the animals. The growth response effect could be as a result of increased food and water intake. Similar findings have been conducted [15-17], and the reported values for ROWs of Sprague dawley rat strains support those of the current data sets. However, age and strains of experimental animals can significantly affect ROW values.

Values are expressed as mean $\pm \mathrm{SD}$ of 5 rats in each group. In each row and each gender $\mathrm{P}$ values $<0.05$ were considered as significant using one way ANOVA followed by Least Significant Difference (LSD). No significant differences were observed in any parameter compared to control (DW). 
Table 1. Effects of oral administration of thymoquinone-rich fraction nanoemulsion (TQRFNE) and distilled water (DW) on mean terminal body weight (bw) and relative organ weights of male and female rats during acute toxicity study

\begin{tabular}{cccccc}
\hline \multirow{2}{*}{ Parameters } & \multicolumn{2}{c}{ Male } & & \multicolumn{2}{c}{ Female } \\
\cline { 2 - 3 } \cline { 5 - 6 } & DW & TQRFNE & DW & TQRFNE \\
\hline Fasted bw (g) & $346 \pm 14$ & $373 \pm 19$ & & $227 \pm 17$ & $265 \pm 20$ \\
Liver & $3.10 \pm 0.18$ & $3.23 \pm 0.22$ & & $2.78 \pm 0.15$ & $2.93 \pm 0.21$ \\
Kidneys & $0.65 \pm 0.05$ & $0.70 \pm 0.07$ & & $0.57 \pm 0.04$ & $0.60 \pm 0.04$ \\
Spleen & $0.23 \pm 0.02$ & $0.24 \pm 0.01$ & & $0.20 \pm 0.02$ & $0.21 \pm 0.01$ \\
Heart & $0.28 \pm 0.01$ & $0.31 \pm 0.04$ & & $0.25 \pm 0.02$ & $0.25 \pm 0.01$ \\
Lungs & $0.54 \pm 0.03$ & $0.54 \pm 0.04$ & & $0.48 \pm 0.04$ & $0.50 \pm 0.07$ \\
Brain & $0.63 \pm 0.05$ & $0.64 \pm 0.07$ & & $0.53 \pm 0.03$ & $0.52 \pm 0.05$ \\
Gonads (Testes/Ovaries) & $0.72 \pm 0.07$ & $0.68 \pm 0.08$ & & $0.65 \pm 0.04$ & $0.65 \pm 0.06$ \\
Stomach (empty) & $0.40 \pm 0.06$ & $0.44 \pm 0.06$ & & $0.36 \pm 0.04$ & $0.37 \pm 0.04$ \\
Gut (empty) & $2.88 \pm 0.35$ & $2.88 \pm 0.34$ & & $2.59 \pm 0.16$ & $2.75 \pm 0.35$ \\
\hline
\end{tabular}

\subsection{Hematology}

There is a correlation of toxicity in hematological, gastrointestinal and cardiovascular adverse effects between animals and humans. In addition, hematological indices in animals are important to determine the toxicity risk since the changes in the blood system have a higher predictive value for human toxicity [18]. In this study, hematological indices were used to assess the toxicity of TQRFNE in Sprague Dawley rats. The hematological parameters of experimental and control rats are presented in Table 2. No significant alterations were seen in the hematological profile of any of the experimental groups studied herein.

Table 2. Effects of oral administration of thymoquinone-rich fraction nanoemulsion (TQRFNE) and distilled water (DW) on hematological parameters of male and female rats

\begin{tabular}{|c|c|c|c|c|}
\hline \multirow{2}{*}{ Parameters } & \multicolumn{2}{|c|}{ Male } & \multicolumn{2}{|c|}{ Female } \\
\hline & DW & TQRFNE & DW & TQRFNE \\
\hline WBC $\left(10^{3} / \mu \mathrm{L}\right)$ & $17.6 \pm 1.7$ & $17.5 \pm 1.3$ & $13.2 \pm 1.1$ & $12.1 \pm 1.2$ \\
\hline RBC $\left(10^{6} / \mu \mathrm{L}\right)$ & $8.0 \pm 0.5$ & $8.0 \pm 0.6$ & $7.0 \pm 0.2$ & $6.8 \pm 0.3$ \\
\hline HGB (g/dL) & $14.3 \pm 0.7$ & $14.6 \pm 1.0$ & $13.4 \pm 0.3$ & $13.9 \pm 0.80$ \\
\hline НСТ \% & $44.6 \pm 2.4$ & $43.3 \pm 1.1$ & $41.9 \pm 2.4$ & $40.5 \pm 3.0$ \\
\hline MCV (FL) & $56.3 \pm 1.1$ & $56.7 \pm 0.7$ & $58.8 \pm 0.7$ & $60.9 \pm 1.8$ \\
\hline МCH (Pg) & $18.0 \pm 0.2$ & $18.2 \pm 0.57$ & $19.4 \pm 0.05$ & $20.2 \pm 0.5$ \\
\hline $\operatorname{MCHC~(g/dL)~}$ & $32.0 \pm 0.4$ & $32.3 \pm 0.45$ & $33.4 \pm 0.37$ & $34.4 \pm 0.8$ \\
\hline $\operatorname{PLT}\left(10^{3} / \mu \mathrm{L}\right)$ & $1108.0 \pm 125$ & $1137.8 \pm 12$ & $1109.2 \pm 55$ & $1292.6 \pm 137$ \\
\hline LYM\# $\left(10^{3} / \mu \mathrm{L}\right)$ & $11.4 \pm 1.2$ & $12.9 \pm 1.2$ & $10.2 \pm 0.6$ & $9.3 \pm 1.5$ \\
\hline PDW (fL) & $9.5 \pm 0.5$ & $9.0 \pm 0.879$ & $9.0 \pm 0.22$ & $8.6 \pm 0.47$ \\
\hline MPV (fL) & $7.7 \pm 0.2$ & $7.4 \pm 0.48$ & $7.6 \pm 0.21$ & $7.4 \pm 0.21$ \\
\hline P-LCR \% & $7.9 \pm 0.2$ & $7.6 \pm 1.0$ & $8.3 \pm 0.92$ & $7.9 \pm 0.47$ \\
\hline
\end{tabular}

Values are expressed as mean \pm SD of five rats in each group. In each row and each gender $\mathrm{P}$ values $<0.05$ were considered as significant using one way ANOVA followed by Least Significant 
Difference (LSD). No significant differences were observed in any parameter compared to control (DW). WBC-white blood cell, RBC-red blood cell, HGB-hemoglobin, HCT-hematocrit, MCV-mean corpuscular volume, MCH-mean corpuscular hemoglobin, MCHC-mean corpuscular hemoglobin concentration, PLT-platelet, LYM-lymphocyte, PDW-platelet distribution width, MPV-mean platelet volume, P-LCR-platelet larger cell ratio.

\subsection{Liver and Kidney Functions Tests}

Liver and kidney function tests are important parameters in determining the safety of functional ingredient or final product [19]. In a toxic environment the blood level of alkaline phosphatase [ALP], alanine amino-tranferase (ALT), asparatae amino-tranferase (AST), gamma glutamyl transpeptidase (GGT), urea (URE), and creatinine (CREA) are known to significantly increase [20,21]. The first four enzymes are reliable indices of liver toxicity and the last two parameters for kidney toxicity [22]. All these parameters in the study (Table 3) showed no appreciable increase in the treated animals except AST in the male rats treated with TQRFNE $(111.0 \pm 13 \mathrm{mM}$ compared to $93.9 \pm 7 \mathrm{mM}$ in control group). AST and ALT are classical enzymes assayed in liver function tests. Increases in the levels of these enzymes above normal are reliable indices of liver toxicity or altered integrity of cellular membrane and cell death [23]. However, AST is not a highly specific indicator for liver injury because it is found in other tissues like the heart, muscles, kidney, and brain, unlike ALT which is found largely in the liver [22]. In addition, the results of the female TQRFNE group were normal compared to control group, so the slightly higher AST may not be considered toxicologically relevant. Hence, this is the first report on the acute oral toxicity study of TQRFNE indicating that it does not have significant toxic effects on the liver and kidneys.

Table 3. Effects of oral administration of thymoquinone-rich fraction nanoemulsion (TQRFNE) and distilled water (DW) on liver and kidney function tests of male and female rats.

\begin{tabular}{cccccc}
\hline \multirow{2}{*}{ Parameters } & \multicolumn{2}{c}{ Male } & & \multicolumn{2}{c}{ Female } \\
\cline { 2 - 3 } \cline { 5 - 6 } & DW & TQRFNE & & DW & TQRFNE \\
\hline ALP $(\mathbf{m M})$ & $123.2 \pm 32$ & $112.9 \pm 13$ & & $71.7 \pm 14$ & $64.2 \pm 14$ \\
ALT(mM) & $40.7 \pm 7$ & $41.2 \pm 4$ & & $40.8 \pm 5$ & $38.3 \pm 2$ \\
AST(mM) & $93.9 \pm 7$ & $111.0 \pm 13^{*}$ & & $89.4 \pm 13$ & $101.7 \pm 10$ \\
GGT(mM) & $7.9 \pm 1$ & $7.6 \pm 1$ & & $7.3 \pm 0.9$ & $7.5 \pm 0.7$ \\
CREA(mM) & $33.3 \pm 3$ & $34.1 \pm 7$ & & $34.3 \pm 5$ & $36.2 \pm 5$ \\
UREA(mM) & $5.7 \pm 0.7$ & $5.4 \pm 0.9$ & & $5.1 \pm 0.3$ & $5.5 \pm 0.8$ \\
\hline
\end{tabular}

Values are expressed as mean $\pm \mathrm{SD}$ of 5 rats per group. In each row and each gender $\mathrm{P}$ values $<0.05$ were considered as significant using one way ANOVA followed by Least Significant Difference (LSD). * denote significance compared to control group (DW). ALP-alkaline phosphatase, ALT-alanine amino-tranferase, AST-asparatae amino-tranferase, GGT-gamma glutamyl transpeptidase, URE-urea, CREA-creatinine. 


\subsection{Histopathology}

The histopathological examinations of the liver tissues from male and female rats were performed to further confirm whether or not the tissues had been damaged (Figures 3 and 4, respectively). No significant histopathological changes in the liver tissues of the experimental animals were observed. Compared to control groups all TQRFNE groups showed normal hepatic architecture with portal triad surrounded by the hepatocytes. Narrow sinusoids open freely into the portal vein. Gross and histopathological examinations further confirmed that the administration of TQRFNE $(20 \mathrm{~mL} / \mathrm{kg})$ for both sexes of Sprague Dawley rats did not cause any evidence of liver tissue damage as compared to control groups. Therefore, the results suggest that the administration of $20 \mathrm{~mL}$ TQRFNE $/ \mathrm{kg}$ is not toxic after an acute exposure.

Figure 3. Hematoxylin and Eosin-stained sections of male liver tissues from control (A) and TQRFNE (B) treated groups. No significant damage was detected in both groups.

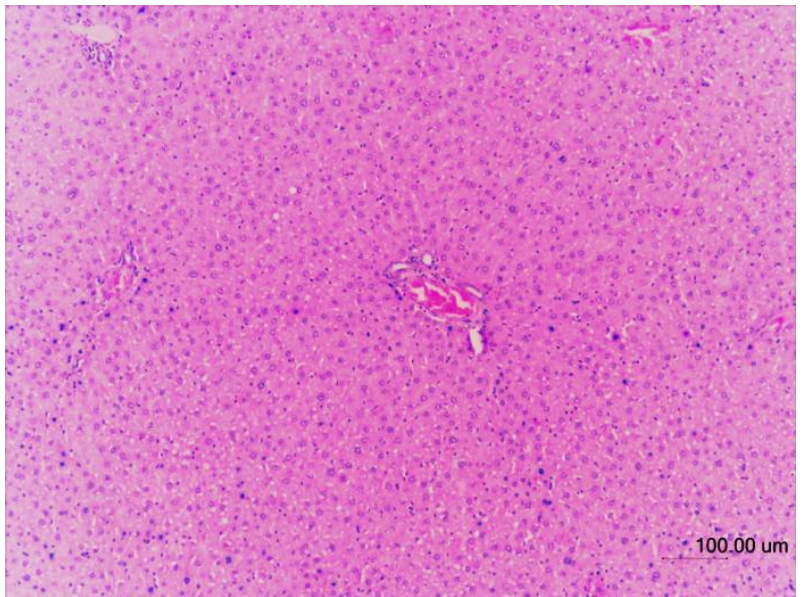

(A)

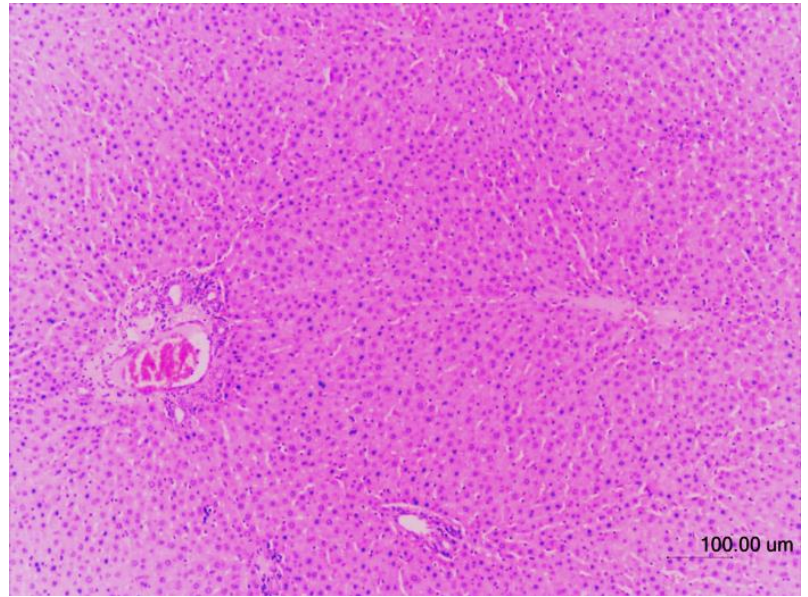

(B)

Figure 4. Hematoxylin and Eosin-stained sections of female liver tissues from control (A) and TQRFNE (B) treated groups. No significant damage was detected in both groups.

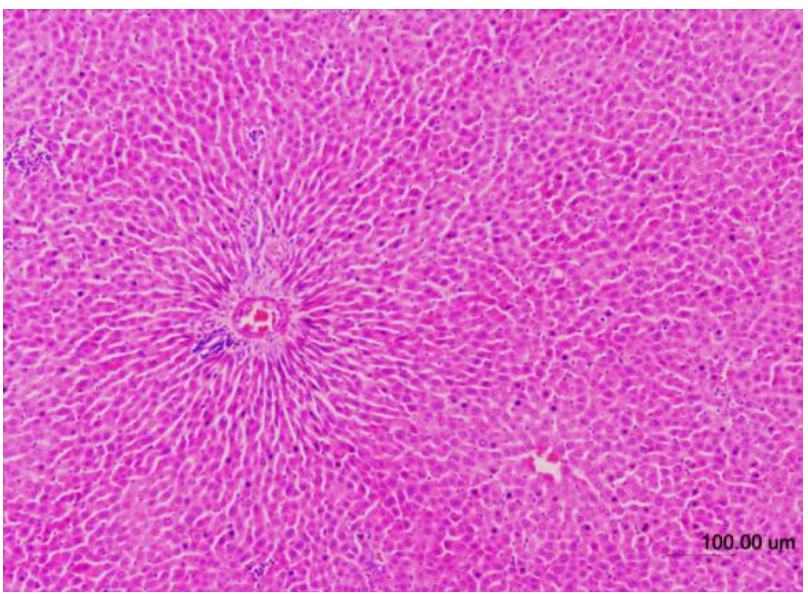

(A)

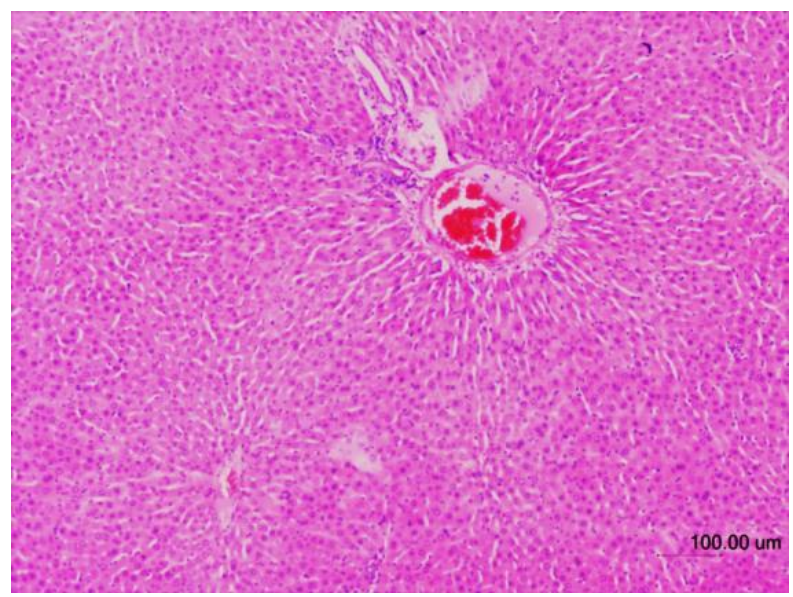

(B) 
Nanoemulsions have a higher solubilization capacity than simple micellar solutions and their thermodynamic stability offers advantages over unstable dispersions such as conventional emulsions [24]. Therefore, TQRFNE may associate with lymph lipoprotein in the enterocytes and gain access to the intestinal lymphatics, effectively bypassing the liver and gaining access to the systemic circulation via the thoracic lymph duct. In addition, we speculated that the presence of TQRF oil in the nanoemulsion would probably enhance oral absorption based on structural similarities to chylomicrons. In an earlier study, a group of 10 mice were given $N$. sativa fixed oil at the dose of $10 \mathrm{~mL} / \mathrm{kg}$ p.o, approximately containing 10 times higher concentration of TQRF compared to that used in the current study. The animals were observed for gross effects and mortality for 15 days. There were no adverse effects or mortality during the observation period following the administration of $N$. sativa fixed oil in mice [25]. In addition, Zaoui, et al. [26] investigated the acute toxicity of the $N$. sativa fixed oil in mice through determination of $\mathrm{LD}_{50}$ values. They found that, $\mathrm{LD}_{50}$ of $N$. sativa fixed oil was $28.8 \mathrm{~mL} / \mathrm{kg}$ bw. p.o. (approximately higher than our TQRF content by 28.8 fold) and $2.06 \mathrm{~mL} / \mathrm{kg}$ bw. i.p., respectively. However, concentrations of TQRF much lower than these were reported to produce functional effects. In fact, TQRF content similar to that in our TQRFNE showed significant improvements in metabolic outcome $[11,27,28]$. In view of the increased solubility and bioavailability of nanoemulsions [29,30], we hypothesized that our TQRFNE, containing a similar amount of TQRF reported to be functionally active, could potentially produce effects at much lower concentrations without the risks of toxicity.

\section{Experimental}

\subsection{Materials}

All the chemicals used in this study were of analytical grade; TQ, Glycerol trioleate (Triolein, TR) and Polysorbate 80 (Tween 80) were purchased from Sigma (Sigma-Aldrich Co., St. Louis, MO, USA). Formalin, cassettes, Haematoxylin and Eosin (H \& E) were purchased from Fisher Scientific (Loughborough, Leicestershire, UK). Clinical chemistry kits for serum biochemistry parameters (ALP, ALT, AST, GGT, URE, and CREA) were purchased from Randox Laboratories Ltd (Crumlin, County Antrim, UK). Double distilled water was used for the preparation of emulsions. Ethanol, xylene, and paraffin were purchased from Merck (Darmstadt, Germany).

\subsection{Production of TQRFNE}

TQRFNE was prepared by homogenizing 5\% of TQRF oil (contains $4.45 \%$ TQ) with 95\% aqueous phase (2\% Tween-80, and 93\% DW) using two sequential homogenization methods. The first method involved homogenizing the solutions in an Ultra-Turrax T25 (IKA, Staufen, Germany) for 3 min at $13000 \mathrm{rpm}$. The second method involved subjecting the previously prepared emulsion to a high-pressure homogenization process using a bench top high-pressure homogenizer (Stansted Fluid Power, Ltd., Essex, UK) at a pressure of 800 bar for five cycles to produce the TQRFNE. To avoid degradation of bioactives, the nanoemulsion was cooled to less than $25{ }^{\circ} \mathrm{C}$ using an ice bath after each homogenization cycle. The final concentration of TQ in the nanoemulsion was determined to be $2.25 \mathrm{mg} / \mathrm{mL}$ (44.5 mg TQ/20 mL TQRFNE). 


\subsection{Experimental Animals}

Male and female Sprague Dawley rats $(n=20,10 \widehat{\delta}$ and 10 क $)$ at 12 weeks of age were used in the study. The animals were purchased from JM Instrument Supply (Selangor, Malaysia). For acclimatization, no more than three animals were housed in each Polypropylene cage, under good laboratory conditions (temperature $25 \pm 3{ }^{\circ} \mathrm{C} ; 55 \pm 5 \%$ relative humidity) with $12 \mathrm{~h}$ dark and light cycle for minimum of 7 days. During this period they had free access to normal rat chow (Specialty Feeds, Memphis, TN, USA) and tap water ad libitum. At the day of dosing, all rats were weighed and examined in detail for physical abnormalities. Group assignments for those judged suitable for testing were generally based on body weight. The study includes control group administered DW and treated group administered TQRFNE. Each group consisted of 10 rats (five males and five females) and body weight ranges at the beginning of the study were 315-337 $\mathrm{g}$ for males and 210-243 $\mathrm{g}$ for females. The present study was conducted in compliance with the test Guidelines of the Organization for Economic Cooperation and Development [31], and was conducted in compliance with FDA Good laboratory practice Regulations [32]. The study protocol was approved by the Animal Care and Use Committee (Approval No. UPM/FPSK//PADS/BR-UUH/00414), Faculty of Medicine and Health Sciences, Universiti Putra Malaysia. Oral administration was selected as the route of administration using a stainless steel feeding needle, with $20 \mathrm{~mL} / \mathrm{kg}$ body weight of DW or TQRFNE. Animals were fasted $12 \mathrm{~h}$ prior to dosing and the food was redistributed $3 \mathrm{~h}$ after the administration [31].

\subsection{Observations}

\subsubsection{Body Weight, and Food and Water Consumption}

Body weight of each rat was taken at the initiation of treatment and once a week thereafter (days 7 and 14) using an electronic balance (Precisa Instrument, Dietikon, Switzerland). The amount of daily water and food consumption was calculated from the difference of initial amounts provided and the remnants for each group. Weekly water and food consumption/rat were also calculated based on the total consumption divided by the number of rats in each group to get the daily consumption of food $(\mathrm{g} / \mathrm{rat} / \mathrm{d})$ and water $(\mathrm{ml} / \mathrm{rat} / \mathrm{d})$.

\subsubsection{Clinical Signs and Necropsy Findings}

Clinical signs and mortalities were monitored continuously for the first hour of administration periodically during the first $24 \mathrm{~h}$, with special emphasis on the first $4 \mathrm{~h}$, and daily thereafter, for a total of 14 days. On the necropsy day (day 15), blood samples ( $3 \mathrm{~mL}$ ) were collected from overnight fasting rats into both anticoagulant-containing (EDTA) and anticoagulant-free (serum blood collection) tubes, by cardiac puncture following light anesthesia. The anti-coagulated blood samples were analyzed immediately for hematology parameters, and the anticoagulant-free blood was used for clinical biochemical study following complete clotting. The serum from the anticoagulant-free tubes was collected by centrifugation at $3500 \mathrm{rpm}$ for $10 \mathrm{~min}$ using centrifuge machine (Hettich EBA 20, Tuttlingen, Germany), and transferred into $1.5 \mathrm{~mL}$ Eppendorf tubes and stored at $-80{ }^{\circ} \mathrm{C}$ until analysis. Following blood collection, rats were killed immediately, and their organs (liver, kidneys, spleen, 
heart, lungs, brain, testes, ovaries, empty stomach and gut) were quickly removed, washed with $0.9 \%$ normal saline and weighed individually. The gross examination (macroscopic analysis) of the target organs of the controls and treated animals was conducted to monitor for any significant change in weight, texture and shape.

\subsubsection{ROW}

Fasted body weight of each animal was measured immediately before sacrificing and the organ weights were measured within 5 min after sacrificing. The ROW (organ to body weight ratio) were calculated as (weight of organ/body weight of rat on the day of sacrifice) $\times 100 \%$ [33].

\subsubsection{Hematology}

The blood was analyzed with KX-21 haematology analyzer (Kobe, Japan). WBC, RBC, HGB, HCT, MCV, MCH, MCHC, PLT, LYM, PDW, MPV and P-LCR counts were measured.

\subsubsection{Liver and Kidney Functions Tests}

For liver and kidney function tests, serum was used to quantify the following parameters: URE, CREA, AST, ALP, and ALT using commercial assay kits (Randox analytical kits) that were based on enzymatic colorimetric methods according to the instructions of the manufacturer on an automated chemistry analyzer, Selectra XL (Vita Scientific, Dieren, The Netherlands). Results were expressed as $\mathrm{mmol} \mathrm{L}{ }^{-1}$.

\subsubsection{Histopathology}

The liver samples collected after sacrificing the animals were washed with $0.9 \%$ normal saline and immediately placed in $10 \%$ formalin before processing. After fixation, the tissues were trimmed at 0.4-0.5 cm thickness and placed in plastic cassettes before they were processed using a tissue processor (Leica TP 1020, Nussloch, Germany). Then, the tissue samples were fixed again in 10\% formalin for $1 \mathrm{~h}$, dehydrated through an ethanol series $(80 \%$ for $1 \times 1 \mathrm{~h}, 95 \%$ for $2 \times 1 \mathrm{~h}$, and $100 \%$ for $3 \times 1 \mathrm{~h})$, washed in xylene $(3 \times 1 \mathrm{~h})$ and paraffin embedded $(2 \times 2 \mathrm{~h})$. Paraffin embedding was done using a Tissue Embedding Console System (Leica EG1160) via a routine method of paraffin embedding procedure. The tissue samples were sectioned at $5 \mu \mathrm{m}$ thicknesses using a microtome (Leica RM2155) and the sections were placed on water bath (Leica H1210) at $35^{\circ} \mathrm{C}$ to $37{ }^{\circ} \mathrm{C}$. Samples were then mounted on glass slides using a hot plate (Leica HI1220) and stained with H \& E stain for histological analysis.

\subsection{Statistical Analysis}

The data were recorded as mean \pm standard deviation and analyzed by SPSS (version 19, SPSS Inc, Chicago, IL). Data were analyzed using one-way ANOVA, followed by Least Significant Difference (LSD). A value of $p<0.05$ was deemed to be statistically significant. 


\section{Conclusions}

In conclusion, TQRFNE was non-toxic by the oral route in male and female Sprague Dawley rats under the conditions of this study at a dose level of $20 \mathrm{~mL} / \mathrm{kg}$. The lack of toxicity of TQRFNE evidenced by the normal levels of hematological, hepatic, and renal key parameters as compared to the control groups suggests a wide margin of safety for its therapeutic doses. In addition, no hepatic toxicity was seen through the histopathology results. However, acute toxicity data sometimes is of limited clinical application since accumulative toxic effect may not be seen in short period with a single dose application. A follow-up study with long-term exposure like sub-acute and chronic toxicity studies may be needed in evaluating the safety profile of TQRFNE.

\section{Acknowledgments}

This study was supported in part by the Research University Grant Scheme, University Putra Malaysia, and nutrigenomic programme. The first author gratefully acknowledges Islamic development bank (IDB) (Jeddah, KSA) for a scholarship provided for PhD.

\section{Conflict of Interest}

The authors declare no conflict of interest.

\section{References}

1. Chaudhary, A.; Nagaich, U.; Gulati, N.; Sharma, V.; Khosa, R.; Partapur, M.U.P. Enhancement of solubilization and bioavailability of poorly soluble drugs by physical and chemical modifications: A recent review. J. Adv. Pharm. Educ. Res. 2012, 2, 32-67.

2. Saraf, S. Applications of novel drug delivery system for herbal formulations. Fitoterapia 2010, 81, 680-689.

3. Goyal, A.; Kumar, S.; Nagpal, M.; Singh, I.; Arora, S. Potential of novel drug delivery systems for herbal drugs. Indian J. Pharm. Educ. Res. 2011, 45, 225.

4. Uhumwangho, M.; Okor, R. Current trends in the production and biomedical applications of liposomes: A review. J. Med. Biomed. Res. 2005, 4, 9-21.

5. Kumar, A.; Ahuja, A.; Ali, J.; Baboota, S. Conundrum and therapeutic potential of curcumin in drug delivery. Crit. Rev. Ther. Drug Carrier Syst. 2010, 27, 279.

6. Youenang Piemi, M.P.; Korner, D.; Benita, S.; Marty, J.P. Positively and negatively charged submicron emulsions for enhanced topical delivery of antifungal drugs. J. Control. Release 1999, 58, 177-187.

7. Ravi Theaj Prakash, U.; Padma, T. Nanoemulsions for drug delivery through different routes. Res. Biotechnol. 2011, 2, 1-13.

8. HHS; FDA; CDER; CBER, Guidance for industry. M3 (R2) Nonclinical Safety Studies for the Conduct of Human Clinical Trials and Marketing Authorization for Pharmaceuticals. In Proceedings of International conference on Harmonisation (ICH), Silver Spring, MD, USA, 21 January 2010. 
9. Barle, E.L.; Looser, R.; Černe, M.; Bechter, R. The value of acute toxicity testing of pharmaceuticals for estimation of human response. Regul. Toxicol. Pharm. 2012, 62, 412-418.

10. Shetty Akhila, J.; Alwar, M. Acute toxicity studies and determination of median lethal dose. Curr. Sci. 2007, 93, 917-920.

11. AL-Naqeeb, G.; Ismail, M. Regulation of apolipoprotein A-1 and apolipoprotein B100 genes by thymoquinone rich fraction and thymoquinone in hepg2 cells. J. Food Lipids 2009, 16, 245-258.

12. Mariod, A.A.; Ibrahim, R.M.; Ismail, M.; Ismail, N. Antioxidant activity and phenolic content of phenolic rich fractions obtained from black cumin (Nigella sativa) seedcake. Food Chem. 2009, 116, 306-312.

13. Randhawa, M.A. Black seed, Nigella sativa, deserves more attention. J. Ayub. Med. Coll. Abbottabad. 2008, 20, 1-2.

14. Ren, H.; Huang, X. Polyacrylate nanoparticles: toxicity or new nanomedicine? Eur. Respir. J. 2010, 36, 218-221.

15. Tauseef Sultan, M.; Butt, M.S.; Anjum, F.M. Safety assessment of black cumin fixed and essential oil in normal Sprague Dawley rats: Serological and hematological indices. Food Chem. Toxicol. 2009, 47, 2768-2775.

16. Kawashima, H.; Toyoda-Ono, Y.; Suwa, Y.; Kiso, Y. Subchronic (13-week) oral toxicity study of dihomo- $\gamma$-linolenic acid (DGLA) oil in rats. Food Chem. Toxicol. 2009, 47, 1280-1286.

17. Han, Z.Z.; Koo, K.H.; Kim, K.H.; Bae, J.S.; Shin, S.H.; Kim, H.S.; Kim, J.H.; Heo, H.S.; Gil, K.H.; Lee, J.Y. Acute and 90-day subchronic toxicity studies of Silk peptide E5K6, in Sprague-Dawley rats. Food Chem. Toxicol. 2011, 49, 2408-2414.

18. Adinortey, M.; Sarfo, J.; Adukpo, G.; Dzotsi, E.; Kusi, S.; Ahmed, M.; Abdul-Gafaru, O. Acute and sub-acute oral toxicity assessment of hydro-alcoholic root extract of Paullinia pinnata on haematological and biochemical parameters. Biol. Med. 2012, 4, 121-125.

19. Farag, R.S.; Mahmoud, E.A.; Basuny, A.M.; Ali, R.F.M. Influence of crude olive leaf juice on rat liver and kidney functions. Int. J. Food Sci. Tech. 2006, 41, 790-798.

20. Meenakshi, V.; Gomathy, S.; Chamundeswari, K. Acute and subchronic oral toxicity of Microcosmus exasperatus Heller, 1878. J. Microbiol. Biotechnol. Res. 2012, 2, 94-98.

21. Mariappan, G.; Sutharson, L.; Srivastav, T.; Kumar, D.; Patangia, U. Pharmacological and toxicological evaluation of some novel 2-substituted 4,5-diphenyl imidazole derivatives. Pharmacologia 2012, 3, 258-266.

22. Adeyemi, O.O.; Akindele, A.J.; Nwumeh, K.I. Acute and subchronic toxicological assessment of Byrsocarpus coccineus Schum. and Thonn. (Connaraceae) aqueous leaf extract. Int. J. Appl. Res. Nat. Prod. 2010, 3, 1-11.

23. Olagunju, J.; Oladunni, S.; Oladimeji, M. Status of phosphatase activities in the liver and kidney of rats treated with isosaline leaf and stem-bark extracts of Harungana madagascariensis (L). Cytobios 2000, 103, 17-24.

24. Shafiq, S.; Shakeel, F.; Talegaonkar, S.; Ahmad, F.J.; Khar, R.K.; Ali, M. Development and bioavailability assessment of ramipril nanoemulsion formulation. Eur. J. Pharm. Biopharm. 2007, 66, 227-243.

25. Zaoui, A.; Cherrah, Y.; Alaoui, K.; Mahassine, N.; Amarouch, H.; Hassar, M. Effects of Nigella sativa fixed oil on blood homeostasis in rat. J. Ethnopharmacol. 2002, 79, 23-26. 
26. Zaoui, A.; Cherrah, Y.; Mahassini, N.; Alaoui, K.; Amarouch, H.; Hassar, M. Acute and chronic toxicity of Nigella sativa fixed oil. Phytomedicine 2002, 9, 69-74.

27. Ismail, M.; Al-Naqeep, G.; Chan, K. Nigella sativa thymoquinone-rich fraction greatly improves plasma antioxidant capacity and expression of antioxidant genes in hypercholesterolemic rats. Free Radic. Biol. Med. 2010, 48, 664-672.

28. Al-Naqeep, G.; Ismail, M.; Yazan, L.S. Effects of thymoquinone rich fraction and thymoquinone on plasma lipoprotein levels and hepatic low density lipoprotein receptor and 3-hydroxy-3methylglutaryl coenzyme A reductase genes expression. J. Funct. Foods 2009, 1, 298-303.

29. Huang, Q.; Yu, H.; Ru, Q. Bioavailability and delivery of nutraceuticals using nanotechnology. J. Food Sci. 2010, 75, R50-R57.

30. McClements, D.J.; Decker, E.A.; Park, Y.; Weiss, J. Structural design principles for delivery of bioactive components in nutraceuticals and functional foods. Crit. Rev. Food Sci. Nutr. 2009, 49, 577-606.

31. OECD. OECD Guideline 425: Acute oral toxicity-Up-and-down procedure. In OECD Guideline for the Testing of Chemicals; Organisation for Economic Co-operation and Development: Paris, France, 2008.

32. FDA. Part 58 - Good laboratory practice for nonclinical laboratory studies. In Code of Federal Regulations Title 21; U.S. Government Printing Office: Washington, DC, USA, 2011.

33. Abdullah, N.R.; Ismail, Z.; Ismail, Z. Acute toxicity of Orthosiphon stamineus Benth standardized extract in Sprague Dawley rats. Phytomedicine 2009, 16, 222-226.

Sample Availability: Samples of the compound are available from the authors.

(C) 2013 by the authors; licensee MDPI, Basel, Switzerland. This article is an open access article distributed under the terms and conditions of the Creative Commons Attribution license (http://creativecommons.org/licenses/by/3.0/). 